\title{
microRNA sponge blocks the tumor-suppressing functions of microRNA-122 in human hepatoma and osteosarcoma cells
}

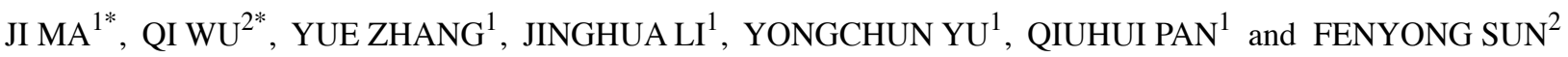 \\ ${ }^{1}$ Central Laboratory and ${ }^{2}$ Department of Medical Laboratory, \\ Shanghai Tenth People's Hospital of Tongji University, Shanghai 200072, P.R. China
}

Received June 12, 2014; Accepted September 9, 2014

DOI: $10.3892 /$ or.2014.3517

\begin{abstract}
RNAs (miRNAs), as gene expression regulators, have been identified to be closely associated with tumorigenesis. Thus a loss-of-function study is more likely to reveal the biological roles of endogenous miRNAs. Genetic knockout, antisense oligonucleotide inhibitors, and miRNA sponge (miR-SP) are usually performed to inhibit the activities of miRNAs of interest. In the present study, we utilized the miR-SP method, which has long-term rather than short-term effects of antisense oligonucleotide inhibitors, to generate a microRNA-122 sponge (miR-122-SP) mediated by lentivirus, and identified its silencing role in the Huh7 hepatoma cell line and U2OS osteosarcoma cell line. The results showed that miR-122-SP effectively sequestered ectopic miR-122 and restored the expression of miR-122 which targets cyclin G1 (CCNG1), Bcl-w and disintegrin and metalloprotease 10. Moreover, miR-122-SP overexpression rescued the effects of ectopic miR-122 on suppressing proliferation, inhibiting cell migration and invasion, arresting cell cycle at G1 phase, and activating caspase-3/7, not only in Huh7 human hepatoma cells, but also in U2OS osteosarcoma cells. miR-122-SP also knocked down endogenous miR-122 expression in Huh7 and
\end{abstract}

Correspondence to: Professor Fenyong Sun, Department of Medical Laboratory, Shanghai Tenth People's Hospital of Tongji University, 301 Middle Yanchang Road, Shanghai 200072, P.R. China

E-mail: sunfenyong2012@gmail.com

Professor Qiuhui Pan, Central Laboratory, Shanghai Tenth People's Hospital of Tongji University, 301 Middle Yanchang Road, Shanghai 200072, P.R. China

E-mail: panqiuhui@263.net

*Contributed equally

Abbreviations: miRNAs, microRNAs; miR-122-SP, miR-122 sponge; 3'-UTR, 3'-untranslated region; miR-SPs, miRNA sponges; miR-122, microRNA-122; HCC, hepatocellular carcinoma; CCNG1, cyclin G1; ADAM10, disintegrin and metalloprotease 10; ADAM17, disintegrin and metalloprotease 17

Key words: microRNA sponge, microRNA-122, cell proliferation, cell cycle, migration, tumorigenesis promoted tumorigenesis in vivo. miR-122-SP therefore is a useful tool that may be utilized to study the functions of miR-122 with regard to liver development and tumorigenesis in vitro and in vivo.

\section{Introduction}

microRNAs (miRNAs) are an abundant class of small noncoding RNAs ( 22 nucleotides) with a critical regulative function $(1,2)$, that reduce mRNA stability and/or suppress translation by perfect or imperfect sequence-complementary binding to the 3'-untranslated region (3'-UTR) or the coding sequences of target mRNAs $(3,4)$. miRNAs have been considered to contribute to the majority of basic biological processes, such as development, differentiation, apoptosis and cell proliferation (5). Their deregulation has also been linked to cancer and other diseases $(6,7)$. Therefore, the in-depth validation of the biological roles of miRNAs is important for understanding pathogenesis and raising new curative therapies.

Several methods for gain- or loss-of-function have been developed to modify the miRNA expression in vitro or in vivo. However, the overexpression of exogenous miRNA (gain-of-function) often leads to neomorphic phenotypes for a strong concentration-dependent interaction between miRNAs and their targets (8). For this reason, a loss-of-function study is more likely to reveal the functions that depend on physiological miRNA levels (9). The miRNA antisense oligonucleotides inhibitors or antagomirs, genetic knockout and miRNA sponge (miR-SPs) methods, have been used to block the activity of miRNA (10-14). It has been reported that miR-SPs, which contain multiple binding sites complementary to a mature miRNA were introduced to sequester endogenous miRNAs and then their functions were suppressed in the in vitro differentiation of neurons (15), mesenchymal stem cells $(16)$, cancer cell xenografts $(17,18)$, bone marrow reconstitutions from hematopoietic stem/progenitor cells (19) and animal models $(20,21)$. Due to the difficult operation of genetic knockouts and short-term effects of antisense oligonucleotide inhibitors, miR-SPs are more suitable to chronically block the activities of endogenous miRNA in vivo.

microRNA-122 (miR-122), a highly abundant liver-specific miRNA that accounts for $70 \%$ of total liver miRNA expression $(22,23)$, has been associated with hepatitis $C$ virus infection and replication $(24,25)$, the regulation of liver metabolism and 
hepatocellular carcinoma (HCC) (26). miR-122 has been identified to downregulate cyclin G1 (CCNG1) $(27,28)$, and thus leads to G1 arrest (29), interrupts the interaction between CCNG1 and p53, and abrogates the p53-mediated inhibition of hepatitis B virus replication (30). Oncogene Bcl-w is also the target of miR-122, and the downregulation of Bcl-w by miR-122 subsequently leads to the reduction of cell viability and activation of caspase-3/7 (28,31). Overexpression of miR-122 affects HCC intrahepatic metastasis by angiogenesis suppression, exerting some of its actions via the regulation of its targets disintegrin and metalloprotease 10 (ADAM10) and ADAM17, which are involved in metastasis $(29,32)$. The loss-of-function study of miR-122 is performed using miR-122 antisense oligonucleotides inhibitors or genetic knockouts, while sponge-mediated miR-122 silencing has yet to be studied in tumor cells.

In the present study, we generated a miR-sponge based on CMV promoter to silence the activity of miR-122 on tumor suppression using lentivirus. Our data showed that the miR-122 sponge (miR-122-SP) effectively blocked the functions of ectopic liver-specific miR-122 by inhibiting cell proliferation, arresting cell cycle, inducing apoptosis and suppressing metastasis, not only in Huh7 hepatoma cells, but also in U2OS osteosarcoma cells. We also found that miR-122-SP efficiently knocked down the expression of endogenous miR-122 in Huh7 cells and promoted xenograft tumor growth. Our studies provide a potential method in which to investigate the miR-122 functions in liver development and tumorigenesis in vitro or in vivo through the application of miR-SPs.

\section{Materials and methods}

Construction of sponge plasmids and reporters. The oligonucleotides with three repetitive sequences, which are complementary to miR-122 with mismatches at positions 9-12, were annealed, ligated, gel purified and cloned into the XhoI/NotI site of a psiCHECK2 vector (Promega, Madison, WI, USA), downstream of the Renilla luciferase gene, to generate the vector $\mathrm{psiCHECK} 2 / \mathrm{miR}-122-\mathrm{SP}$. We constructed the GFP reporters using the same annealing method and subcloned the sequences into the 3'-UTR of the EGFP in a pEGFP vector with a CMV promoter.

Cell culture and stable cell line production. The Huh7 cell line (American Type Culture Collection, Manassas, VA, USA) was maintained at $37^{\circ} \mathrm{C}, 5 \% \mathrm{CO}_{2}$ and in DMEM/high glucose (Invitrogen Life Technologies, Carlsbad, CA USA) with 10\% fetal bovine serum (FBS). The U2OS cell line (American Type Culture Collection) was maintained at $37^{\circ} \mathrm{C}, 5 \% \mathrm{CO}_{2}$ and in RPMI-1640 supplemented with 10\% FBS (both from Invitrogen Life Technologies) in 25-ml culture flasks. For the miR-122-SP stable overexpression, the Huh7 or U2OS cells were infected with lentivirus that expressed miR-122-SP (Genchem \& Genpharm Co., Ltd., Jiangsu, China) or its negative control at $30-50 \%$ confluence. The monoclonal population of the stably infected cells was selected by a limiting dilution assay using the GFP marker.

Luciferase and GFP reporter assay. For the luciferase reporter assay, $293 \mathrm{~T}$ cells $\left(5 \times 10^{4}\right.$ cells/well) were seeded in 24 -well plates $24 \mathrm{~h}$ prior to transfection. The cells were co-transfected with $0.5 \mu \mathrm{g}$ of the psiCHECK2/miR-122-SP plasmids together with $30 \mathrm{nM}$ of miR-122 mimics or $30 \mathrm{nM}$ of the negative control (both from GenePharma, Shanghai, China) using Lipofectamine 2000 (Invitrogen Life Technologies). Firefly and Renilla luciferase activities were measured by using the dual-luciferase reporter assay (Promega) $48 \mathrm{~h}$ after transfection. The firefly luciferase activity was normalized to the Renilla luciferase activity. For the GFP reporter assay, U2OS cells $\left(5 \times 10^{4}\right.$ cells/well) seeded in 24 -well plates were co-transfected with $0.5 \mu \mathrm{g}$ of the $\mathrm{pEGFP} / \mathrm{miR}-122-\mathrm{SP}$ plasmids together with $30 \mathrm{nM}$ of miR-122 mimics or $30 \mathrm{nM}$ of the negative control (both from GenePharma) using Lipofectamine 2000 (Invitrogen Life Technologies). GFP expression was examined by a microscope after $48 \mathrm{~h}$ of transfection.

RT-qPCR analysis. The total RNA from tissue samples and cell lines was extracted using TRIzol reagent (Invitrogen Life Technologies) following the manufacturer's instructions. The RNA concentrations and quality were determined with a spectrophotometer (Eppendorf AG, Hamburg, Germany) and gel analysis. RT-qPCR was performed using SYBR Premix Ex Taq (Takara Bio, Inc., Shiga, Japan) and Applied Biosystems 7900 Real-Time PCR System (Applied Biosystems, Foster City, CA, USA) supplied with its analytical software. The forward and reverse primer pairs were designed for CCNG1, Bcl-w, and ADAM10 as described previously $(28,29)$. The primer pair for the 18SrRNA Q-PCR was as follows: forward, 5'-CCTGGATACCGCAGCTAGGA-3' and reverse, 5'-GCG GCGCAATACGAATGCCCC-3'. The expression of mature miR-122 was assayed using the miRCURY LNA ${ }^{\mathrm{TM}}$ Universal RT microRNA PCR Universal cDNA synthesis kit with specific primers (both from Exiqon, Vedbaek, Denmark) on cell lines. Reverse transcription reaction was carried out starting from $1 \mu \mathrm{g}$ of total RNA using real-time PCR was performed according to the miRCURY LNA ${ }^{\mathrm{TM}}$ SYBR-Green master mix kit (Exiqon) protocol. The $\Delta \Delta \mathrm{Ct}$ method for relative quantification was used to determine the gene expression.

Western blot analysis. The cells transfected with the indicated vectors or chemicals were subjected to western blotting with anti-CCNG1 $(1: 1,000)$, anti-Bcl-w $(1: 1,000)$ (both from Santa Cruz Biotechnology, Inc., Santa Cruz, CA, USA), antiADAM10 (1:1,000; eBioscience, Inc., San Diego, CA, USA) and actin (1:1,500; Santa Cruz Biotechnology, Inc.) antibodies, respectively. The cells were harvested and lysed in $0.5 \mathrm{ml}$ of lysis buffer (10 mM Tris-HCl, pH 7.6, $5 \mathrm{mM}$ EDTA, $50 \mathrm{mM}$ $\mathrm{NaCl}, 30 \mathrm{mM}$ sodium pyrophosphate, $50 \mathrm{mM} \mathrm{NaF}, 0.1 \mathrm{mM}$ $\mathrm{Na}_{3} \mathrm{VO}_{4}, 1 \%$ Triton X-100, 1 mM PMSF and protease inhibitors. Proteins $(20 \mu \mathrm{g})$ were processed for SDS-PAGE, which was performed on $10 \%$ gels. The proteins were electrophoretically transferred to PVDF membranes (Millipore, Billerica, MA, USA) and incubated with the primary antibodies followed by incubation with an HRP-conjugated secondary antibody (Santa Cruz Biotechnology, Inc.). After washing with TBS, the bound antibodies were visualized by enhanced chemiluminescence (Pierce Biotechnology, Inc., Rockford, IL,USA) and recorded on X-ray film.

Cell proliferation assay. Ctrl-lentivirus (Ctrl-LV) and miR-122-SP-LV cells $\left(2.5 \times 10^{5}\right.$ cells/well $)$ were transfected 
with $30 \mathrm{nM}$ miR-122 mimics or $30 \mathrm{nM}$ negative control (GenePharma) using Lipofectamine 2000 (Invitrogen Life Technologies) as per the manufacturer's instructions in 6-well plates. The transfected cells $\left(1 \times 10^{3}\right.$ cells/well $)$ were then seeded in 96-well plates. The MTT assay was performed on day 1,3,5 and 6. Absorbance of the samples was measured with a spectrophotometer reader at $490 \mathrm{~nm}$, and each assay was performed in triplicate.

Colony formation assay. Each cell line transfected with synthesized oligonucleotides or vectors was seeded in 12-well plates (200 cells/well), incubated for 7-10 days to allow colony growth, and the colonies were stained with crystal violet. The colonies containing $\geq 50$ cells were counted. The plating efficiency was calculated by dividing the average number of the colonies/dish by the number of the cells plated. The survival fractions were calculated by normalizing to the plating efficiency of the control groups.

Wound-healing scratch assay. For the wound-healing scratch assay, $2.5 \times 10^{5} \mathrm{Ctrl}-\mathrm{LV}$ and miR-122-SP-LV cells transfected with $30 \mathrm{nM}$ of miR-122 mimics or negative controls were seeded in 12-well plates. After $24 \mathrm{~h}$, a linear wound was made by scratching across the bottom of the dish on the monolayer of the confluent cells using a sterile p-200 pipette tip. The cells were rinsed gently with phosphate-buffered saline (PBS) and cultivated in the corresponding medium without serum supplemented with $0.5 \%$ FBS. The same area of the gap was taken at X100 magnifications using a microscope equipped with a digital camera (Olympus, Centre Valley, PA, USA) at 0 and $24 \mathrm{~h}$ after the scratching, and then quantified using ImageJ software (http://rsbweb.nih.gov/ij/). The difference between the initial and final areas was calculated.

Cell Matrigel invasion assay. For the invasion assay, $1 \times 10^{5}$ Ctrl-LV and miR-122-SP-LV cells transfected with $30 \mathrm{nM}$ of miR-122 mimics or negative controls were seeded in a Matrigel-coated chamber with $8.0 \mu \mathrm{M}$ pores (Corning Costar, Corning, NY, USA). The cells were seeded in serumfree media in the upper chamber, and translocated towards complete growth media for $36 \mathrm{~h}$. The lower compartment of the chamber was fixed in $1.5 \%$ glutaraldehyde for $1 \mathrm{~h}$, stained with $0.1 \%$ violet crystal dye for $15 \mathrm{~min}$, and washed twice with distilled water. The stained cells were viewed under a light microscope (Olympus).

Cell cycle analysis. To examine the effect of miR-122-SP on the blocking functions of miR-122 in cell cycle arrest, the Ctrl-LV and miR-122-SP-LV cells were transfected with $30 \mathrm{nM}$ of miR-122 mimics or negative controls and then collected after $48 \mathrm{~h}$ and analyzed for DNA content by flow cytometry.

Caspase-3/7 activity analysis. The Ctrl-LV and miR-122-SP$\mathrm{LV}$ cells transfected with $30 \mathrm{nM}$ of miR-122 mimics or negative controls were seeded in 96 -well plates $\left(5 \times 10^{3}\right.$ cells/well). After $24 \mathrm{~h}$ of incubation at $37^{\circ} \mathrm{C}$, caspase-3/7 substrates (Promega) were added according to the manufacturer's instructions and incubated for $1 \mathrm{~h}$ at $37^{\circ} \mathrm{C}$ in the dark. The relative light intensity was measured in each well using a microplate luminometer.
Xenograft tumor model. Huh7-Ctrl-LV or Huh7-miR-122SP-LV cells $\left(1 \times 10^{5}\right)$ were inoculated subcutaneously into the right flanks of female athymic nude nu/nu mice (3-4 weeks old), respectively. The volume of the implanted tumor was measured every 4 days with a vernier caliper, using the formula: $\mathrm{V}=\mathrm{Lx} \mathrm{W}^{2} / 2$; where $\mathrm{V}$, volume $\left(\mathrm{mm}^{3}\right)$; L, biggest diameter (mm); W, smallest diameter (mm). The mice were subsequently sacrificed via cervical dislocation and the tumors were weighed 4 weeks after inoculation.

Study approval. All animal procedures were undertaken according to the United Kingdom Co-ordinating Committee on Cancer Research (UKCCCR) Guidelines for the Welfare of Animals in Experimental Neoplasia and were approved by the Central Laboratory, Shanghai Tenth People's Hospital of Tongji University (Shanghai, China).

Statistical analysis. Data were presented as means \pm SD. The Student's t-test was applied to determine the differences between sample means obtained from at least three experiments. Statistical analyses were performed using Excel software. All tests of statistical significance were two-sided. $\mathrm{P}<0.05$ was considered to indicate statistical significance.

\section{Results}

Construction of miR-122-SP. To generate a miR-122-SP, three repetitive sequences, complementary to miR-122 with mismatches at positions 9-12 for enhanced stability, were introduced into the 3'-UTR of Renilla luciferase in a psiCHECK2 vector (Fig. 1A and B). We first co-transfected HEK293T cells with psiCHECK2/miR-122-SP plasmids and miR-122 mimics and assayed the activity of the Renilla luciferase $48 \mathrm{~h}$ after the transfection. The data showed that miR-122 decreased the activity of luciferase by directly binding to the miR-122-SP sites in the 3'-UTR region downstream of Renilla luciferase, while the luciferase activities in the cells co-transfected with miR-122 mimics and psiCHECK2 vector, or microRNA negative control (NC) and miR-122-SP, were not altered (Fig. 1D). To identify the effect of sponge, we sub-cloned miR-122-SP sites into the 3'-UTR of the EGFP in a pEGFP vector with a CMV promoter (Fig. 1C). We co-transfected this pEGFP/ miR-122-SP with miR-122 mimics into U2OS cells, and found that the GFP was silenced in the U2OS cells with miR-122 overexpression (Fig. 1E). These results showed that the ectopic miR-122 directly bound to the miR-122-SP, which subsequently resulted in the decrease of reporter gene expression, suggesting that the construction of miR-122-SP was successful.

MiR-122-SP reduces miR-122 expression and upregulates the target genes of miR-122. As a liver-specific microRNA, miR-122 expression was confirmed to be downregulated in the HCC of rodent and human (33). We detected miR-122 expression in different types of cell lines by RT-qPCR. No endogenous expression of miR-122 was observed in Hep3B hepatoma cells and U2OS osteosarcoma cells, but a low expression of miR-122 was identified in Huh7 cells (Fig. 2A). To study the miR-122-SP effects on blocking miR-122 functions, we chose Huh7 hepatoma cells with a low expression of miR-122 and U2OS non-hepatoma cells without any expression of miR-122 


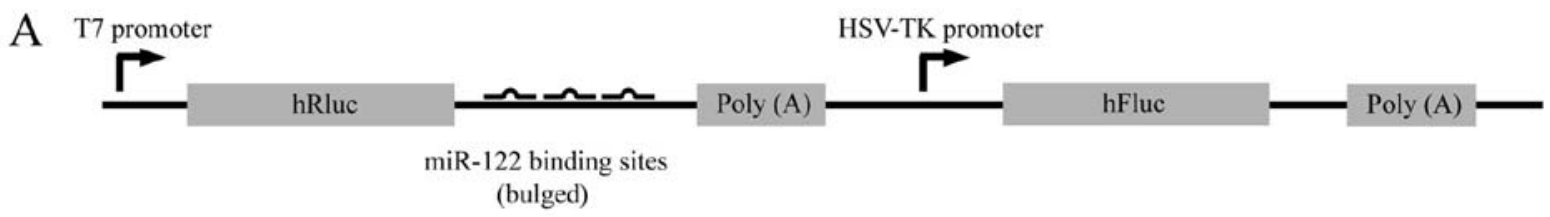

B

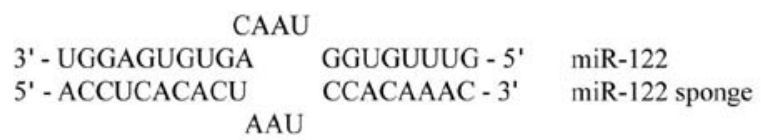

C
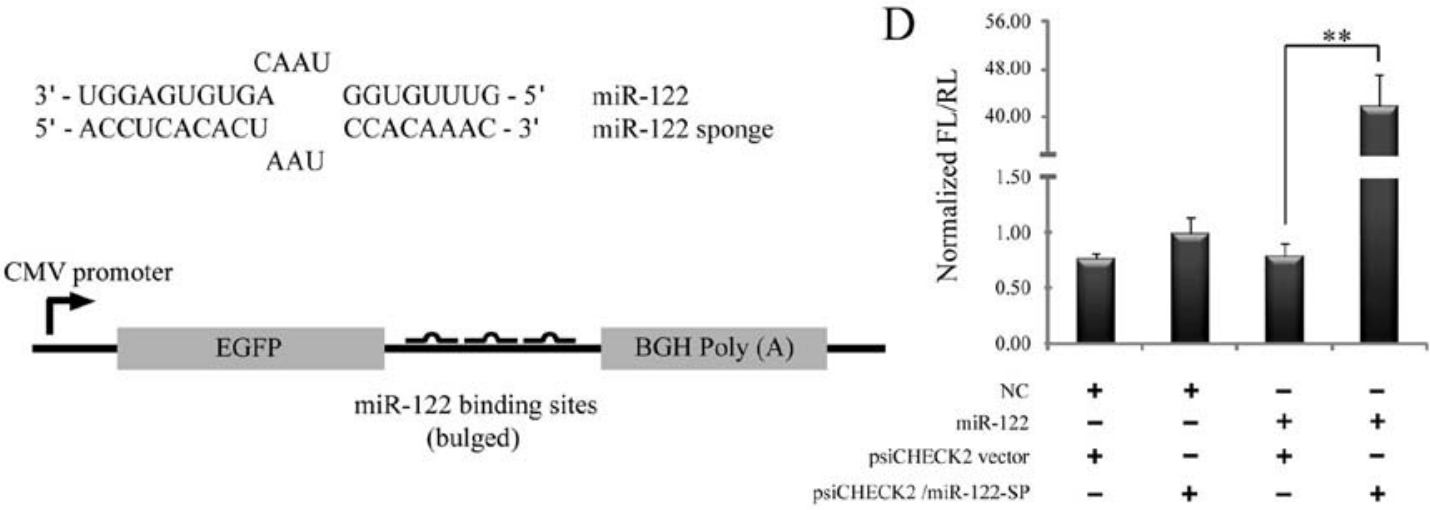

E
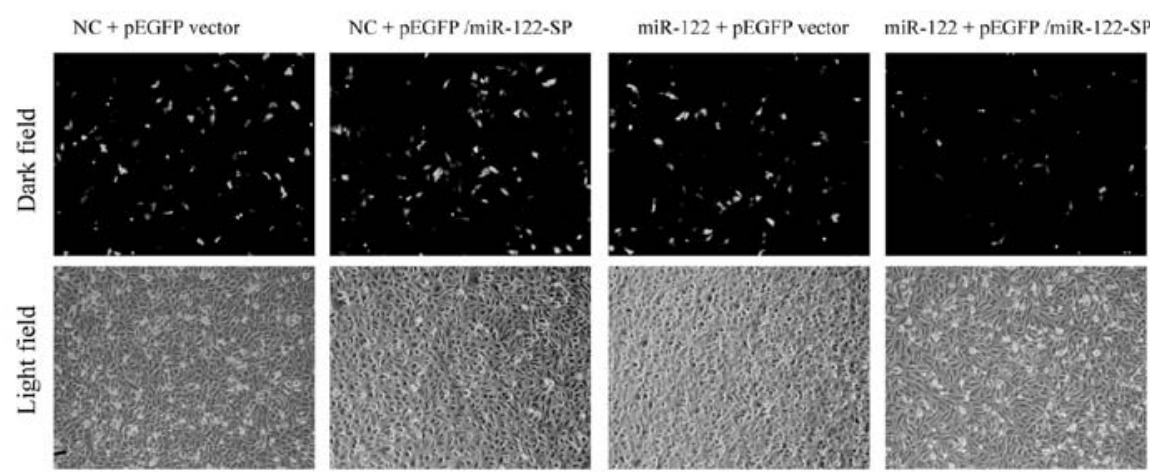

Figure 1. Construction of microRNA-122 sponge (miR-122-SP). (A) Design of psiCHECK2/miR-122-SP. Three miR-122 bulged binding sites were inserted into the 3'-untranslated region (3'-UTR) of Renilla luciferase reporter gene driven by the T7 promoter followed with firefly luciferase reporter gene driven by HSV-TK promoter. (B) The imperfect pairing between miR-122 and its sponge with bulged binding sites. (C) Design of pEGFP/miR-122-SP. The same binding site sequences such as those in (A) were inserted into the 3'-UTR of GFP driven by the CMV promoter. (D) The activity of Renilla luciferase (RL) was downregulated by miR-122-SP depending on miR-122. HEK293T cells were co-transfected with psiCHECK2/miR-122-SP and miR-122 mimics. The luciferase activities are not altered. The bars indicate that firefly luciferase (FL) activities normalized to Renilla from the psiCHECK-2 vector. Each experiment was repeated at least three times, and each sample was assayed in triplicate. ${ }^{* *} \mathrm{P}<0.01$ compared with the control. (E) The expression of GFP was downregulated by miR-122-SP depending on miR-122 in U2OS cells co-transfected with pEGFP/miR-122-SP and miR-122 mimics.

as cell models. Due to the low efficiency and short-term effect of the transient transfection of plasmid, we used a lentivirusmediated expression system to express miR-122-SP in Huh7 and U2OS cells in the same manner as indicated in Fig. 1C. The RT-qPCR results showed that stable overexpression of miR-122-SP led to a decrease of ectopic miR-122 expression in Huh7 and U2OS cells, as well as the downregulation of endogenous miR-122 expression in Huh7 cells (Fig. 2A). These results indicated that the lentivirus-mediated miR-122-SP expression was able to efficiently reduce the endogenous or ectopic expression of miR-122.

As a tumor suppressor, miR-122 has been identified to downregulate the expression of CCNG1 (involved in the cell cycle), Bcl-w (involved in apoptosis) and ADAM10 (involved in migration). To investigate whether miR-122-SP decreased the expression of these genes, we examined the mRNA and protein levels in Huh7 and U2OS cells. As expected, the mRNA levels of the Huh7 and U2OS cells were inhibited by ectopic miR-122, while the cells with miR-122-SP expression completely rescued the expression of these three genes (Fig. 2B). The western blot results revealed the same effects of miR-122-SP on reducing the protein levels of CCNG1, Bcl-w and ADAM10 in the Huh7 and U2OS cells (Fig. 2C). However, the mRNA and protein levels of CCNG1, Bcl-w and ADAM10 were not significantly altered by miR-122-SP expression without ectopic miR-122. This result may be due to the low expression of miR-122 in Huh7 that could not obviously inhibit the expression of the three genes. These results suggested that miR-122-SP would clearly be able to restore the expression of CCNG1, Bcl-w and ADAM10 that was downregulated by ectopic miR-122.

miR-122-SP blocks the functions of miR-122 in cell proliferation. miR-122 has been identified to significantly inhibit tumor cell proliferation. To identify whether miR-122-SP suppressed the functions of miR-122 in cell proliferation, Ctrl-LV or miR-122-SP-LV cells were transfected with miR-122 mimics or the negative control. We found that Huh7 or U2OS Ctrl-LV cell proliferation was significantly inhibited by the ectopic miR-122 expression through the MTT assay, while proliferations of the Huh7 or U2OS miR-122-SP-LV cells transfected with miR-122 


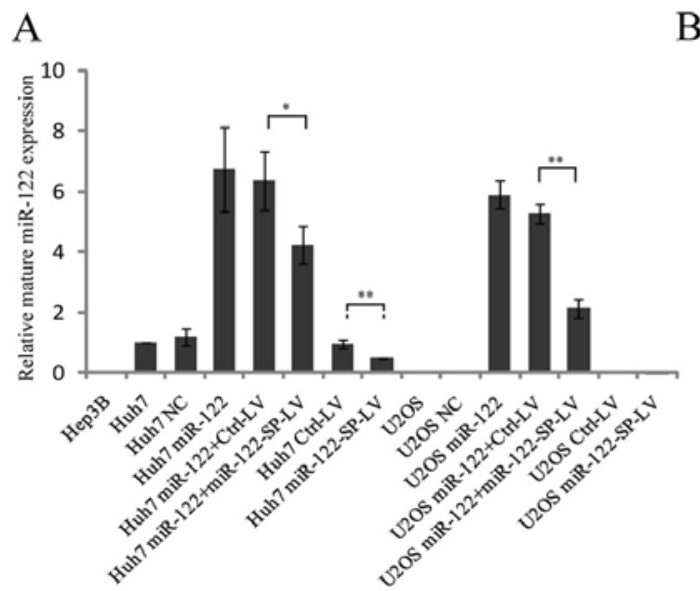

B

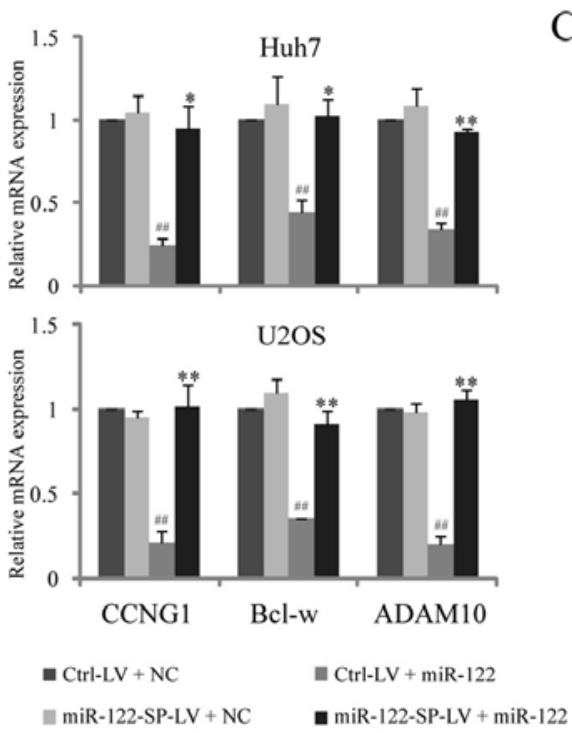

$\mathrm{C}$
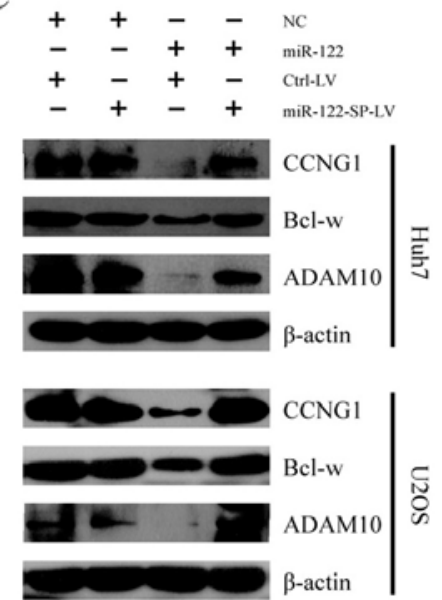

III $\mathrm{miR}-122-\mathrm{SP}-\mathrm{LV}+\mathrm{NC}$

a miR-122-SP-LV + miR-122

Figure 2. microRNA-122 sponge (miR-122-SP) reduces miR-122 expression and restores the expression of target genes downregulated by miR-122.(A) miR-122 expression in Hep3B, Huh7 and U2OS cancer cells was detected by RT-qPCR. Huh7 and U2OS cells infected by Ctrl-lentivirus (Ctrl-LV) or miR-122-SPlentivirus (miR-122-SP-LV) were transfected with $30 \mathrm{nM}$ miR-122 mimics or negative control (NC), and then collected after $24 \mathrm{~h}$ for total RNA isolation. ${ }^{*} \mathrm{P}<0.05$ and ${ }^{* *} \mathrm{P}<0.01$ compared with NC. (B) The mRNA expression of cyclin G1 (CCNG1), Bcl-w and disintegrin and metalloprotease 10 (ADAM10) was determined by RT-qPCR. Data are shown as $2^{-\Delta \Delta C t}$ values. ${ }^{\# / t} \mathrm{P}<0.001$ compared with Ctrl-LV $+\mathrm{NC}$. ${ }^{*} \mathrm{P}<0.05$ and ${ }^{* *} \mathrm{P}<0.001$ compared with Ctrl-LV + miR-122. (C) The protein levels of CCNG1, Bcl-w and ADAM10 were examined by western blot analysis. $\beta$-actin was a loading control. Data present at least three independent experiments with similar results.
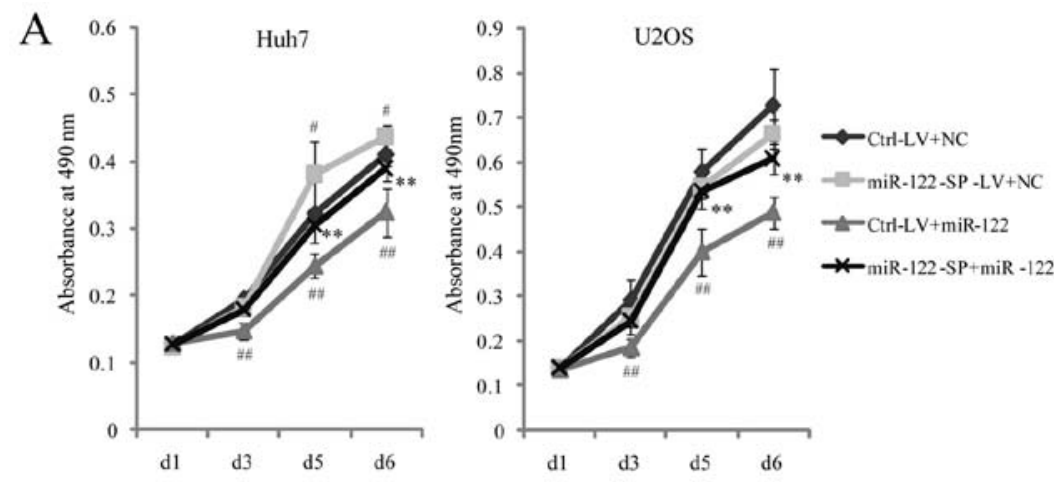

C

B
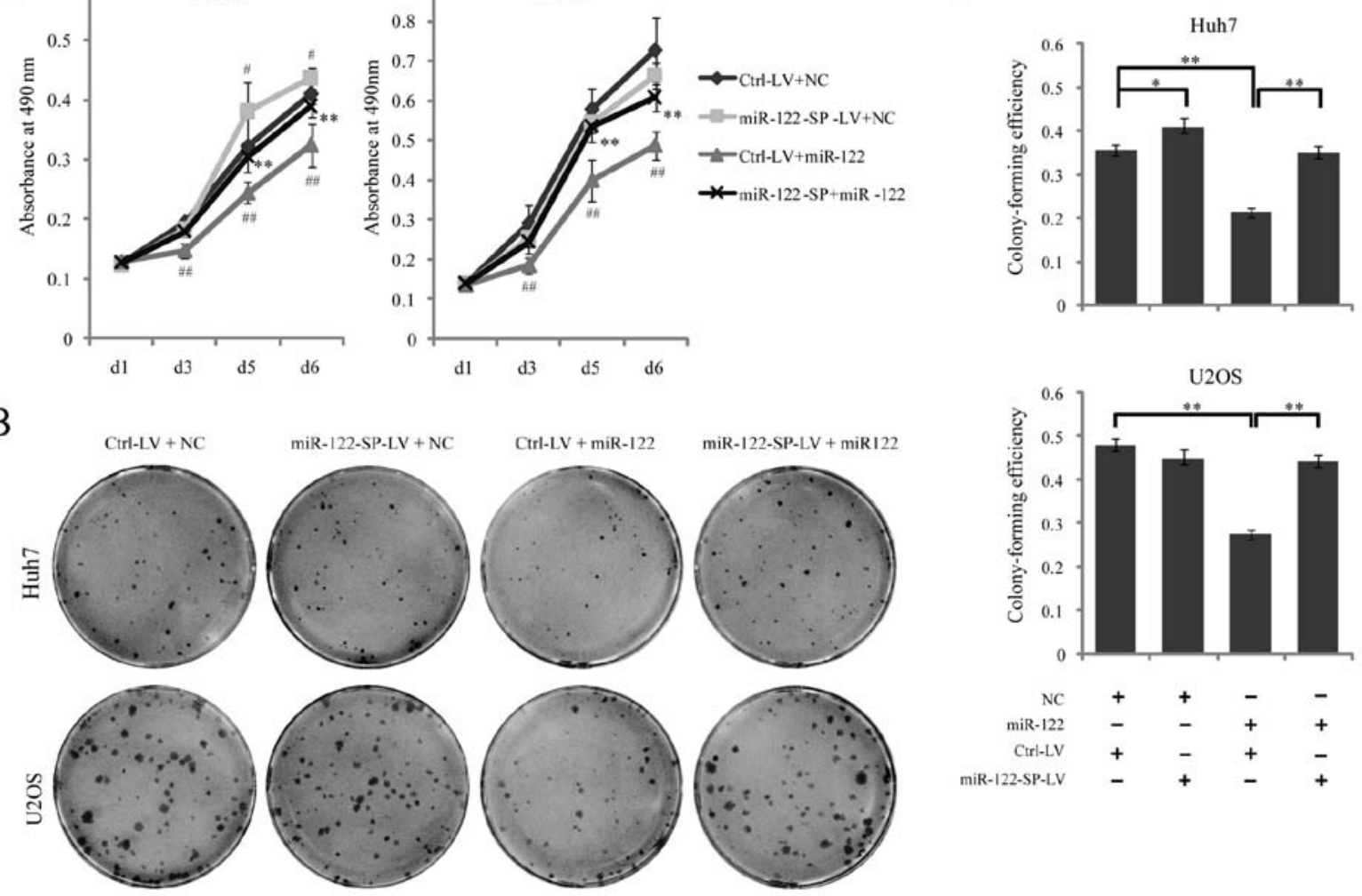

Figure 3. microRNA-122 sponge (miR-122-SP) blocks the functions of miR-122 in cell proliferation. (A) Ctrl-lentivirus (Ctrl-LV) and miR-122-SP-LV cells transfected with $30 \mathrm{nM}$ miR-122 mimics or negative control (NC) were subjected to MTT analysis at different time periods. Data present at least three independent experiments. ${ }^{\# \#} \mathrm{P}<0.001$ compared with Ctrl-LV + NC. ${ }^{* *} \mathrm{P}<0.01$ compared with Ctrl-LV + miR-122. (B and C) The results of colony formation assay. Representative images for colony enumeration are shown in (B). The data of colony formation efficiency of (C) miR-122-SP or control cells. Data present at least three independent experiments. ${ }^{* *} \mathrm{P}<0.01$.

were not obviously altered (Fig. 3A). Fig. 3C also showed that miR-122 could suppressed the colony-forming efficiency of Huh7 and U2OS cells, while miR-122-SP with miR-122 mimics had no effect on colony formation (Fig. 3B and C). In 

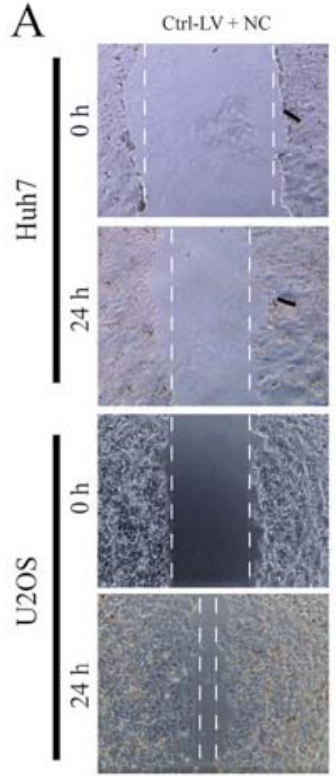

C
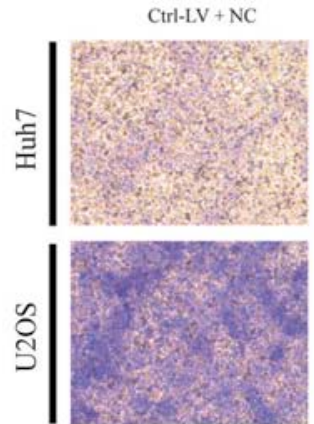
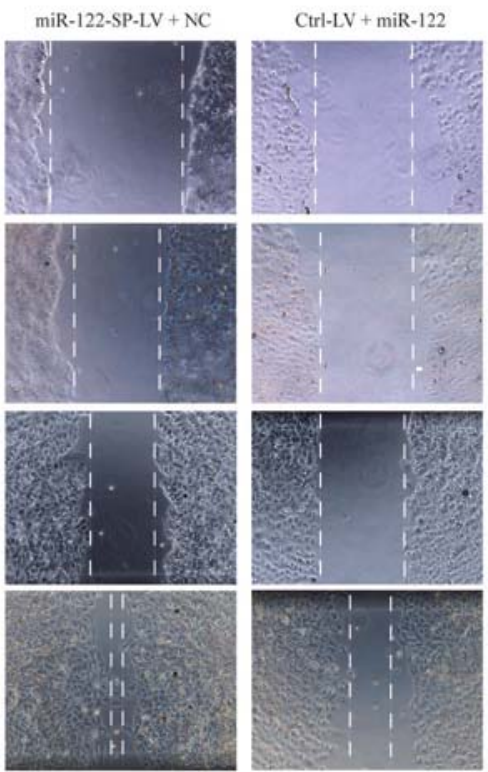

miR-122-SP-LV + NC
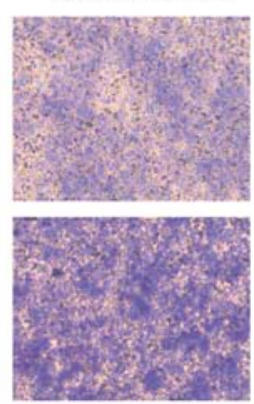
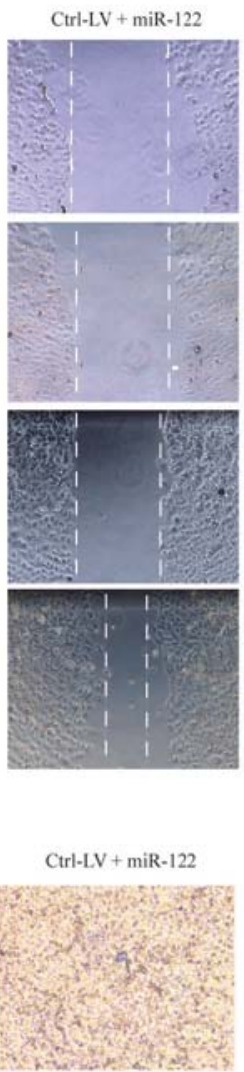

miR-122-SP-LV + miR-122
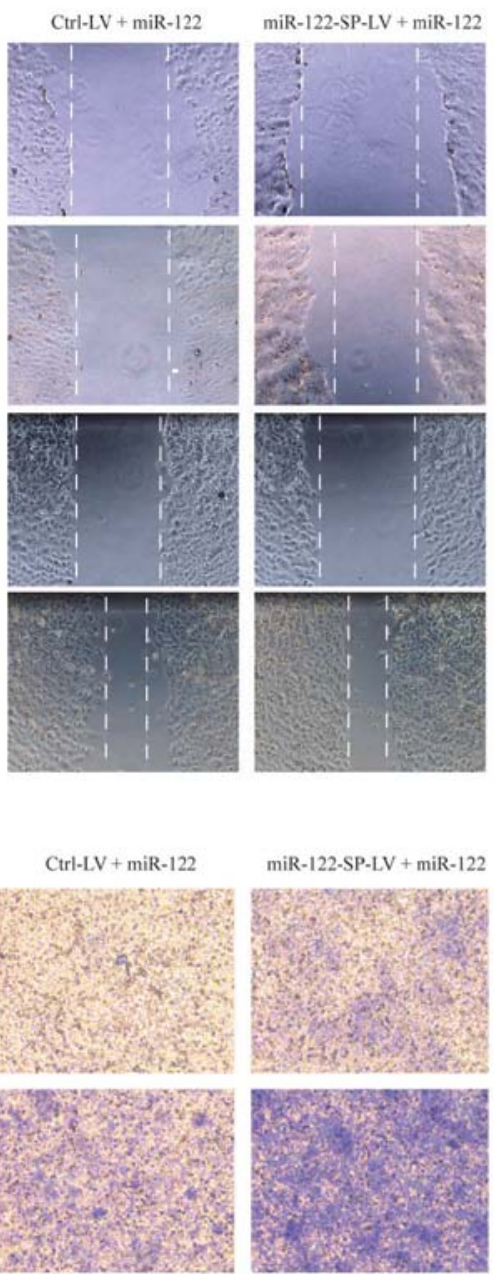

B
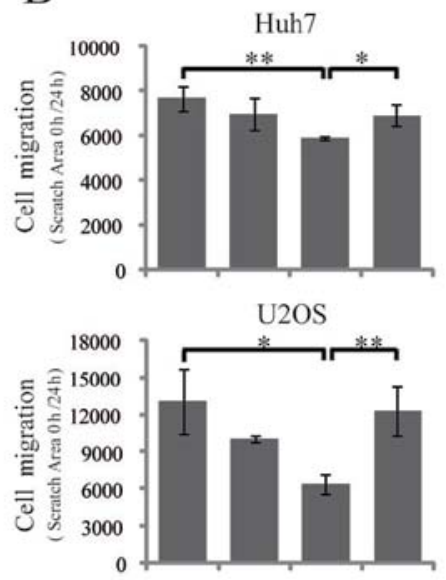

$\mathrm{D}$
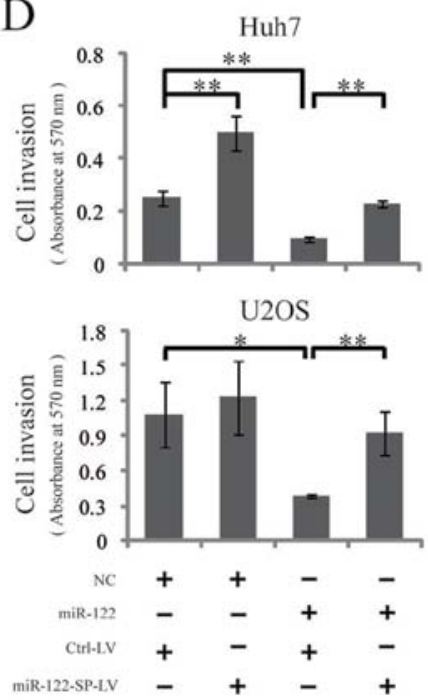

Figure 4. microRNA-122 sponge (miR-122-SP) blocks the functions of miR-122 in cell migration and invasion. Ctrl-lentivirus (Ctrl-LV) and miR-122-SP-LV cells transfected with $30 \mathrm{nM}$ miR-122 mimics or negative control (NC) were subjected to a wound-healing scratch assay and Matrigel invasion assay. (A) After transfection for $24 \mathrm{~h}$, a line was drawn across the bottom of the dish on confluent cells and re-cultured in medium with $0.5 \%$ fetal bovine serum (FBS). Phase-contrast microscopy images were captured of the same wounded area at 0 and $24 \mathrm{~h}$ after the line. (B) Cell migration quantification of images in (A) was assessed by scratch area at $0 \mathrm{~h}$ normalized to $24 \mathrm{~h}$. Data present at least three independent experiments with similar results. ${ }^{*} \mathrm{P}<0.05$ and ${ }^{* *} \mathrm{P}<0.01$. $(\mathrm{C}) \mathrm{The}$ treated cells were re-plated in serum-free medium layered onto the top chambers and invaded to the bottom chamber containing serum-supplemented medium for $36 \mathrm{~h}$ at $37^{\circ} \mathrm{C}$. (D) Cell invasion quantification of images in (C) was assessed by detecting the absorbance at $570 \mathrm{~nm}$. Data present at least three independent experiments with similar results. ${ }^{*} \mathrm{P}<0.05$ and ${ }^{* *} \mathrm{P}<0.01$.

accordance with the decrease of endogenous miR-122 expression by miR-122-SP in Huh7, we also found that miR-122-SP promoted cell proliferation and increased the cell colony formation ability of Huh7 cells (Fig. 3A), which was caused by the downregulation of endogenous miR-122 in Huh7-mediated by miR-122-SP. These results indicated that miR-122-SP was able to rescue the effects of liver-specific miR-122 on suppressing proliferation, not only in Huh7 human hepatoma cells, but also in U2OS osteosarcoma cells.

miR-122-SP blocks the functions of miR-122 in cell migration and invasion. To study the effects of miR-122-SP on inhibiting the functions of miR-122 in cell migration, we conducted wound-healing scratch experiments to assess cell migration. Ctrl-LV or miR-122-SP-LV cells were transfected with miR-122 mimics or negative controls on 12-well plates. After culturing for $24 \mathrm{~h}$ in medium with $10 \% \mathrm{FBS}$, the confluent culture monolayer of these cells was scratched and cultured in medium with $0.5 \%$ FBS for another $24 \mathrm{~h}$. The same wounded area/well was examined by phase-contrast microscopy after scratching at 0 and $24 \mathrm{~h}$. Transfection with miR-122 mimics significantly inhibited the migration of U2OS and Huh7 cells, while the miR-122-SP expression blocked the functions of miR-122 by inhibiting cell migration (Fig. 4A and B). Subsequently, we subjected a Matrigel invasion assay to define the effect of miR-122-SP on blocking the functions of miR-122 in suppressing cell invasion. As expected, the results showed that miR-122 decreased the number of invading cells in Huh7 and U2OS, while this decrease induced by miR-122 was significantly inhibited by miR-122-SP, suggesting a suppressor role of miR-122-SP for miR-122 in cell migration and invasion (Fig. 4C and D).

miR-122-SP blocks the functions of $m i R-122$ in the regulation of cell cycle and caspase-3/7 activity. CCNG1 and Bcl-w were identified as the targets of miR-122, and ectopic miR-122 led 

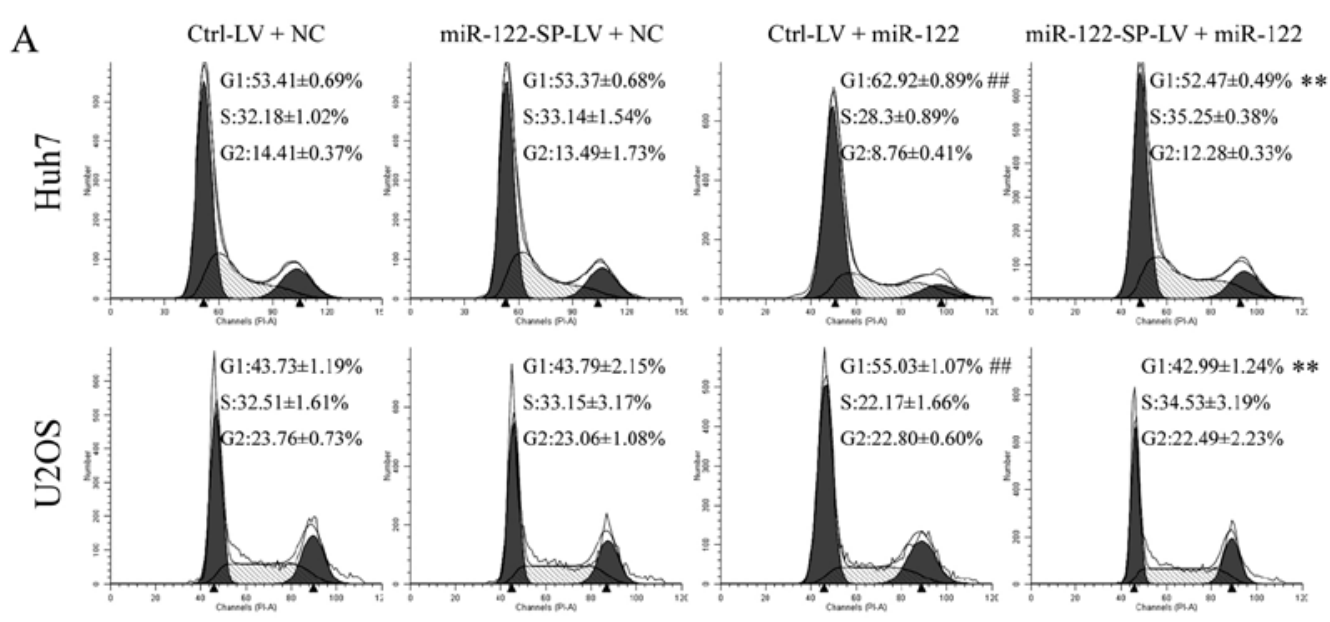

B

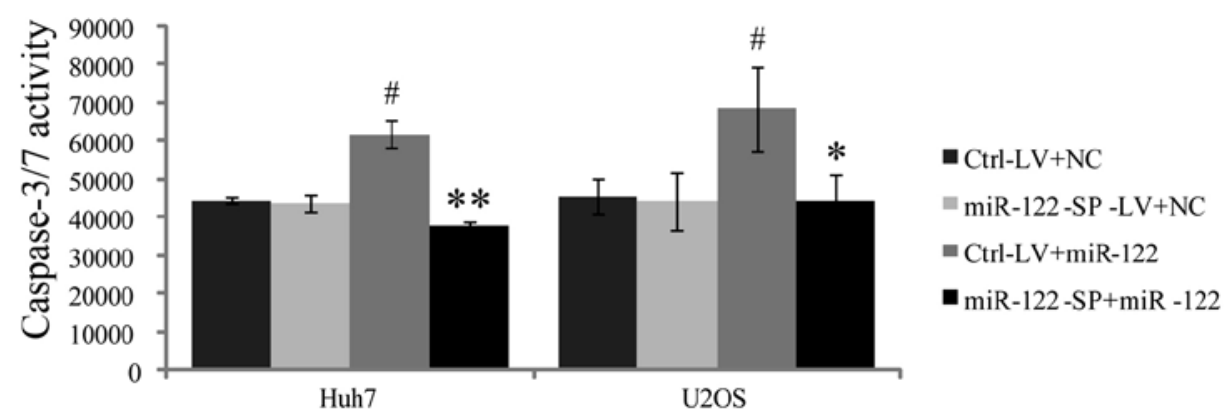

Figure 5. microRNA-122 sponge (miR-122-SP) blocks the functions of miR-122 in the regulation of the cell cycle and caspase-3/7 activity. (A) Ctrl-lentivirus (Ctrl-LV) and miR-122-SP-LV cells transfected with $30 \mathrm{nM}$ miR-122 mimics or negative control (NC) were harvested after $48 \mathrm{~h}$ and analyzed for DNA content by flow cytometry. Data represent at least three independent experiments with similar results. Values are presented as means \pm standard deviation (SD), $\mathrm{n}=3$. Compared with NC, ${ }^{\# \#} \mathrm{P}<0.01$ compared with Ctrl-LV + NC. ${ }^{* *} \mathrm{P}<0.01$ compared with Ctrl-LV + miR-122. (B) Caspase-3/7 activities in Ctrl-LV and miR-122-SP-LV cells were measured after transfection with $30 \mathrm{nM}$ miR-122 mimics or negative control after $48 \mathrm{~h}$. Compared with $\mathrm{NC}$, "P $<0.05$, compared with Ctrl-LV + NC. ${ }^{*} \mathrm{P}<0.05$ and ${ }^{* *} \mathrm{P}<0.01$ compared with Ctrl-LV + miR-122.

A

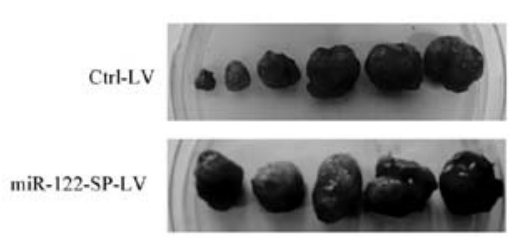

B

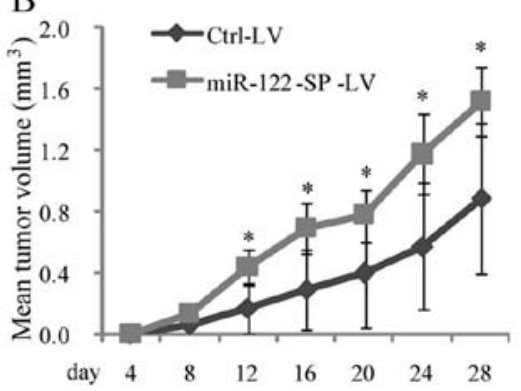

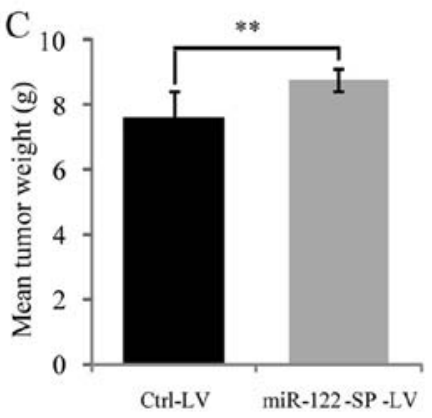

Figure 6. Knockdown of microRNA-122 (miR-122) function by miR-122 sponge (miR-122-SP) promotes tumorigenesis. (A) Huh7-Ctrl-lentivirus (Ctrl-LV) or Huh7-miR-122-SP-LV cells were inoculated subcutaneously into nude mice $(\mathrm{n}=6)$. The mice were sacrificed and the tumors were weighed 28 days after inoculation. (B and C) Increasing miR-122-SP expression in Huh7 cells exacerbates the tumor burden. (B) The average volume of tumors developed in nude mice is shown as mean \pm standard deviation (SD). $\mathrm{P}<0.05$, compared with Ctrl-LV. (C) The average tumor weight is indicated as mean \pm standard deviation (SD). ${ }^{* *} \mathrm{P}<0.01$, compared with negative control.

to $\mathrm{G} 1$ arrest of cancer cells and increased caspase-3/7 activity. Thus, we hypothesized that miR-122-SP affected the cell cycle and the activity of caspase-3/7 through miR-122. To confirm this, we harvested Ctrl-LV and miR-122-SP-LV cells $48 \mathrm{~h}$ after the cells were transfected with miR-122 mimics or negative controls to analyze the cell cycle distribution by FACS. Our results showed that there was a significant increase in the population of cells at G1 phase between the miR-122 expressing and the control cells (62.92 and 53.41\% in Huh7 cells, and 55.03 and
$43.73 \%$ in U2OS cells). However, miR-122-SP overexpression restored the distribution of G1 phase from 62.92 to $52.47 \%$ in Huh7 cells and 55.03 to $42.99 \%$ in U2OS (Fig. 5A). These results demonstrated that miR-122-SP reduced the effects of miR-122 on arresting cell cycle at G1 phase. Subsequently, we detected the caspase-3/7 activity of the Ctrl-LV and miR-122-SP-LV cells that were transfected with or without miR-122. The caspase-3/7 activity was inhibited by miR-122; however, it was rescued by the overexpression of miR-122-SP in Huh7 and 
U2OS cells (Fig. 5B). This suggested that miR-122-SP was able to block the functions of miR-122 in promoting cell apoptosis.

miR-122-SP blocks the functions of miR-122 in tumor suppression. Although, a low expression of miR-122 was detected in Huh7 cells, miR-122-SP was able to reduce the endogenous miR-122 expression and block the functions of miR-122 in inhibiting cell proliferation. To determine whether miR-122-SP blocks activities of miR-122 in suppressing tumorigenesis in vivo, Huh7 cells infected with Ctrl-LV or miR-122-SP-LV were subcutaneously inoculated into nude mice. The mean volume of xenograft tumors formed in the presence of miR122-SP was larger than that of control (Fig. 6A and B). At the end of the experimental period, the mean weight of tumors with miR-122-SP overexpression was higher than that of the control (Fig. 6C). Thus, these data indicate that miR-122-SP blocks the functions of miR-122 in inhibiting tumorigenesis in vivo.

\section{Discussion}

To investigate the functions of miRNAs, the loss-of-function study is considered more reliable than that of gain-of-function for its blocking endogenous miRNAs activity (34). Several methods were utilized to study miRNA loss-of-function, including genetic knockouts, antisense oligonucleotide inhibitors $(12,35,36)$ and sponges $(9)$. The use of modified antisense oligonucleotide inhibitors, also designated as antagomirs or locked nucleic acids (LNAs), was the first method to block miRNA activity in animal cell cultures $(12,36,37)$. However, antagomirs, as well as LNAs did not reach some tissues, such as the central nervous system, or the cells in a brain due to the blood-brain barrier $(12,38,39)$. Moreover, they only provide short-term downregulation of the miRNA targets and block single miRNA rather than whole miRNA families (14), suggesting that antagomirs and LNAs are not suitable for generating transgenic animal models.

Compared to antisense oligonucleotide inhibitors, miR-SPs offer more advantages for many in vivo research applications. Firstly, sponges mediated by a lentiviral vector can stably and chronically block the miRNA activity in difficult-to-transfect cell lines or cells in vivo. Secondly, a single miR-SP can block the activities of the whole family of miRNAs sharing highly similar seed sequences while locating in multiple distant loci (9). Furthermore, a reporter gene or a selectable marker in sponge facilitates the introduction of sponge for tracing, screening and sorting cells with miRNAs. A tissue-specific or drug-inducible promoter in a sponge, such as the tet-on and tet-off system, is more useful to study the functions of many important miRNAs in development without leading to transgenic model death.

Accumulating evidence has demonstrated that miR-122 is downregulated in chronic hepatitis B (CHB) and HCC of human $(30,33)$, suggesting the possible relationship between hepatocarcinogenesis and liver-specific microRNA. Currently, studies on miR-122 function in tumorigenesis is based on the ectopic expression of miR-122 in hepatoma cells in vitro. Although miR-122 has been confirmed to suppress tumorigenic properties of Huh7 cells, which express miR-122 at a lower level, by transfecion of anti-miR-122 oligonucleotides in vitro (29), the roles of endogenous of miR-122 in hepatoma cells in vivo have not been reported. Thus, we designed lentivirus-mediated miR-122-SP, which can be used to silence the activities of miR-122 in vitro and in vivo, to confirm or identify the roles of miR-122 in liver development and hepatocarcinogenesis. In this study, we confirmed that ectopic miR-122 downregulated the expression of CCNG1, Bcl-w and ADAM10, inhibited cell proliferation and migration, arrested the cell cycle at G1 phase, and induced cell apoptosis in Huh7 hepatoma cells in vitro. These effects of miR-122 on suppressing tumorigenesis be efficiently blocked by miR122 -SP. In addition, we may found similar results in U2OS osteosarcoma cells, that miR-122-SP was able to silence the functions of ectopic miR-122 in suppressing tumorigenic properties. These results suggest that miR-122-SP is an effective tool for loss-of-function research in vitro, and raise the potential of miR-122 as small molecular medicine for other types of cancer therapy.

In this study, we found that endogenous miR-122 in Huh7 cells infected with miR-122-SP-LV was evidently downregulated (Fig. 2A) compared to cells infected with control lentivirus, and the effects of endogenous miR-122 on cell proliferation (Fig. 3) and migration (Fig. 4C and D) were efficiently blocked. However, the target expression of miR-122 and the effects of miR-122 on cell cycle and apoptosis were not significantly rescued by miR-122-SP, which we hypothesize are due to a lower expression of miR-122 in Huh7 cells. An increased expression level of miR-122 in Huh7, we identified a significant differences in target expression, cell proliferation, migration and apoptosis between the miR-122-SP-LV and control cells. Furthermore, miR-122-SP was able to obviously promote xenograft tumor growth by silencing the endogenous miR-122 in Huh7 (Fig. 6). These data suggest that miR-122-SP$\mathrm{LV}$ is a useful tool for in vivo research. Using this system, we can examine more functions of miR-122 in liver development and hepatocarcinogenesis.

In conclusion, our design of miR-122-SP can successfully silence miR-122 effects on inhibiting tumorigenesis in vitro and in vivo, suggesting that miR-122-SP has potential roles for generating liver cancer models with a lower expression of miR-122 to investigate the miR-122 functions in liver development and diseases.

\section{Acknowledgements}

This study was supported by the National Natural Science Foundation of China (grant no. 81301704 to J.M.; grant no. 81071524 to F.S.) (URL: http://www.nsfc.gov.cn/Portal0/ default152.htm)

\section{References}

1. Calin GA and Croce CM: MicroRNA signatures in human cancers. Nat Rev Cancer 6: 857-866, 2006.

2. Garzon R, Fabbri M, Cimmino A, Calin GA and Croce CM: MicroRNA expression and function in cancer. Trends Mol Med 12: 580-587, 2006.

3. Zeng Y: Principles of micro-RNA production and maturation. Oncogene 25: 6156-6162, 2006.

4. Valencia-Sanchez MA, Liu J, Hannon GJ and Parker R: Control of translation and mRNA degradation by miRNAs and siRNAs. Genes Dev 20: 515-524, 2006. 
5. Ahmed FE: Role of miRNA in carcinogenesis and biomarker selection: a methodological view. Expert Rev Mol Diagn 7: 569-603, 2007

6. Chen CZ: MicroRNAs as oncogenes and tumor suppressors. N Engl J Med 353: 1768-1771, 2005.

7. Croce CM: Oncogenes and cancer. N Engl J Med 358: 502-511, 2008.

8. Bushati N and Cohen SM: microRNA functions. Annu Rev Cell Dev Biol 23: 175-205, 2007.

9. Ebert MS and Sharp PA: MicroRNA sponges: progress and possibilities. RNA 16: 2043-2050,2010.

10. Brown BD and Naldini L: Exploiting and antagonizing microRNA regulation for therapeutic and experimental applications. Nat Rev Genet 10: 578-585, 2009.

11. Kloosterman WP, Lagendijk AK, Ketting RF, Moulton JD and Plasterk RH: Targeted inhibition of miRNA maturation with morpholinos reveals a role for miR-375 in pancreatic islet development. PLoS Biol 5: e203, 2007.

12. Krützfeldt J,Rajewsky N, Braich R, et al: Silencing of microRNAs in vivo with 'antagomirs'. Nature 438: 685-689, 2005.

13. Park CY, Choi YS and McManus MT: Analysis of microRNA knockouts in mice. Hum Mol Genet 19: R169-R175, 2010.

14. Ebert MS, Neilson JR and Sharp PA: MicroRNA sponges: competitive inhibitors of small RNAs in mammalian cells. Nat Methods 4: 721-726, 2007.

15. Barbato C, Ruberti F, Pieri M, et al: MicroRNA-92 modulates $\mathrm{K}(+) \mathrm{Cl}(-)$ co-transporter $\mathrm{KCC} 2$ expression in cerebellar granule neurons. J Neurochem 113: 591-600, 2010.

16. Huang J, Zhao L, Xing L and Chen D: MicroRNA-204 regulates Runx 2 protein expression and mesenchymal progenitor cell differentiation. Stem Cells 28: 357-364, 2010.

17. Bonci D, Coppola V, Musumeci M, et al: The miR-15a-miR-16-1 cluster controls prostate cancer by targeting multiple oncogenic activities. Nat Med 14: 1271-1277, 2008.

18. Valastyan S, Reinhardt F, Benaich N, et al: A pleiotropically acting microRNA, miR-31, inhibits breast cancer metastasis. Cell 137: 1032-1046, 2009

19. Papapetrou EP, Korkola JE and Sadelain M: A genetic strategy for single and combinatorial analysis of miRNA function in mammalian hematopoietic stem cells. Stem Cells 28: 287-296, 2010.

20. Gentner B, Schira G, Giustacchini A, et al: Stable knockdown of microRNA in vivo by lentiviral vectors. Nat Methods 6: 63-66, 2009.

21. Loya CM, Lu CS, Van Vactor D and Fulga TA: Transgenic microRNA inhibition with spatiotemporal specificity in intact organisms. Nat Methods 6: 897-903, 2009.

22. Lagos-Quintana M, Rauhut R, Yalcin A, Meyer J, Lendeckel W and Tuschl T: Identification of tissue-specific microRNAs from mouse. Curr Biol 12: 735-739, 2002.

23. Landgraf $\mathrm{P}$, Rusu $\mathrm{M}$, Sheridan $\mathrm{R}$, et al: A mammalian microRNA expression atlas based on small RNA library sequencing. Cell 129: 1401-1414, 2007.
24. Jopling CL, Norman KL and Sarnow P: Positive and negative modulation of viral and cellular mRNAs by liver-specific microRNA miR-122. Cold Spring Harb Symp Quant Biol 71: 369-376, 2006

25. Jopling CL: Regulation of hepatitis $C$ virus by microRNA-122. Biochem Soc Trans 36: 1220-1223, 2008

26. Girard M, Jacquemin E, Munnich A, Lyonnet S and Henrion-Caude A: miR-122, a paradigm for the role of microRNAs in the liver. J Hepatol 48: 648-656, 2008.

27. Gramantieri L, Ferracin M, Fornari F, et al: Cyclin G1 is a target of miR-122a, a microRNA frequently down-regulated in human hepatocellular carcinoma. Cancer Res 67: 6092-6099, 2007.

28. Ma L, Liu J, Shen J, et al: Expression of miR-122 mediated by adenoviral vector induces apoptosis and cell cycle arrest of cancer cells. Cancer Biol Ther 9: 554-561, 2010.

29. Bai S, Nasser MW, Wang B, et al: MicroRNA-122 inhibits tumorigenic properties of hepatocellular carcinoma cells and sensitizes these cells to sorafenib. J Biol Chem 284: 32015-32027, 2009.

30. Wang S, Qiu L, Yan X, et al: Loss of microRNA 122 expression in patients with hepatitis B enhances hepatitis B virus replication through cyclin G(1)-modulated P53 activity. Hepatology 55: 730-741, 2012.

31. Lin CJ, Gong HY, Tseng HC, Wang WL and Wu JL: miR-122 targets an anti-apoptotic gene, Bcl-w, in human hepatocellular carcinoma cell lines. Biochem Biophys Res Commun 375: 315-320, 2008.

32. Tsai WC, Hsu PW, Lai TC, et al: MicroRNA-122, a tumor suppressor microRNA that regulates intrahepatic metastasis of hepatocellular carcinoma. Hepatology 49: 1571-1582, 2009.

33. Kutay H, Bai S, Datta J, et al: Downregulation of miR-122 in the rodent and human hepatocellular carcinomas. J Cell Biochem 99: 671-678, 2006

34. Sahu SC, O'Donnell MW Jr and Sprando RL: Interactive toxicity of usnic acid and lipopolysaccharides in human liver HepG2 cells. J Appl Toxicol 32: 739-749, 2012.

35. Meister G, Landthaler M, Dorsett Y and Tuschl T: Sequencespecific inhibition of microRNA- and siRNA-induced RNA silencing. RNA 10: 544-550, 2004.

36. Ørom UA, Kauppinen S and Lund AH: LNA-modified oligonucleotides mediate specific inhibition of microRNA function. Gene 372: 137-141, 2006.

37. Davis S, Lollo B, Freier S and Esau C: Improved targeting of miRNA with antisense oligonucleotides. Nucleic Acids Res 34: 2294-2304, 2006.

38. Obad S, dos Santos CO, Petri A, et al: Silencing of microRNA families by seed-targeting tiny LNAs. Nat Genet 43: 371-378, 2011.

39. Otaegi G, Pollock A and Sun T: An optimized sponge for microrna miR-9 affects spinal motor neuron development in vivo. Front Neurosci 5: 146, 2012. 